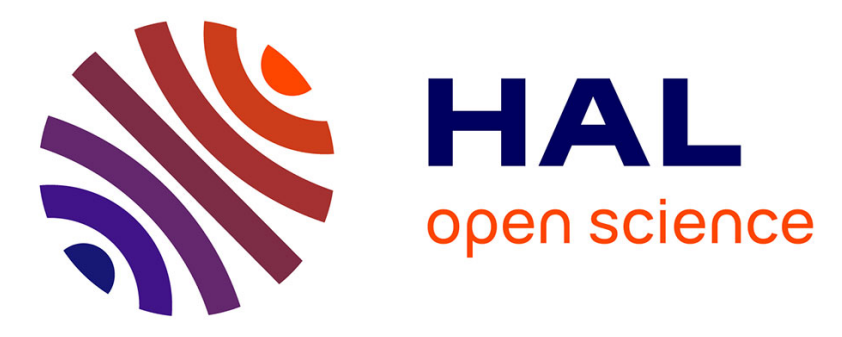

\title{
Mechanism of activation of plant monogalactosyldiacylglycerol synthase 1 (MGD1) by phosphatidylglycerol
}

Milène Nitenberg, Olga N Makshakova, Joana Rocha, Serge Perez, Eric Maréchal, Maryse Block, Agnès Girard-Egrot, Christelle Breton

\section{To cite this version:}

Milène Nitenberg, Olga N Makshakova, Joana Rocha, Serge Perez, Eric Maréchal, et al.. Mechanism of activation of plant monogalactosyldiacylglycerol synthase 1 (MGD1) by phosphatidylglycerol. Glycobiology, 2020, 30 (6), pp.409-419. 10.1093/glycob/cwz106 . hal-02517538

\section{HAL Id: hal-02517538 \\ https://hal.science/hal-02517538}

Submitted on 24 Sep 2020

HAL is a multi-disciplinary open access archive for the deposit and dissemination of scientific research documents, whether they are published or not. The documents may come from teaching and research institutions in France or abroad, or from public or private research centers.
L'archive ouverte pluridisciplinaire HAL, est destinée au dépôt et à la diffusion de documents scientifiques de niveau recherche, publiés ou non, émanant des établissements d'enseignement et de recherche français ou étrangers, des laboratoires publics ou privés.

\section{(c)(1)}

Distributed under a Creative Commons Attribution| 4.0 International License 


\section{Mechanism of activation of plant monogalactosyldiacylglycerol synthase 1 (MGD1) by phosphatidylglycerol}

\section{Milène Nitenberg ${ }^{1}$, Olga Makshakova ${ }^{2}$, Joana Rocha ${ }^{1}$, Serge Perez $^{1}$, Éric Maréchal ${ }^{3}$, Maryse. A. Block ${ }^{3}$, Agnès Girard-Egrot ${ }^{4 *}$, Christelle Breton ${ }^{1 *}$}

${ }^{1}$ University Grenoble Alpes, CNRS, Centre de Recherches sur les Macromolécules Végétales (CERMAV), Grenoble, France.

${ }^{2}$ Kazan Institute of Biochemistry and Biophysics, FRC Kazan Scientific Center of RAS, Kazan, Russia

${ }^{3}$ Laboratoire de Physiologie Cellulaire et Végétale (LPCV), UMR 5168, CNRS, CEA, INRA, University Grenoble Alpes, Grenoble France.

${ }^{4}$ University of Lyon, Institut de Chimie et Biochimie Moléculaires et Supramoléculaires (ICBMS), UMR 5246, CNRS, Univ. Lyon 1 5246, GEMBAS team, Villeurbanne, France.

*co-corresponding authors

Correspondence: CERMAV-CNRS, CS40700, 38041 Grenoble cedex 9, France. E-mail: christelle.breton@.cermav.cnrs.fr

Correspondence: University of Lyon 1, ICBMS, UMR CNRS 5246, GEMBAS team, Bât. Lederer, 1 rue Victor Grignard, 69622 Villeurbanne cedex, France. E-mail: agnes.girard-egrot@univlyon1.fr

Running title: MGD1 activation by phosphatidylglycerol 


\begin{abstract}
Abbreviations:
AFM, atomic force microscopy; DAG, diacylglycerol; DGDG, digalactosyldiacylglycerol; IEM, inner envelope membrane; LB, Langmuir-Blodgett; MGD1, monogalactosyldiacylglycerol synthase type 1; MGDG, monogalactosyldiacylglycerol; MIP, maximum insertion pressure; PA, phosphatidic acid; PG, phosphatidylglycerol; SQDG, sulfoquinovosyldiacylglycerol; UDP-Gal, uridine 5'-diphosphate- $\alpha$-D-galactose; $\Pi$, surface pressure; $\Pi_{e}$, equilibrium surface pressure; $\Pi_{\mathrm{i}}$, initial surface pressure.
\end{abstract}




\begin{abstract}
Mono- and digalactosyldiacylglycerol (MGDG and DGDG) are essential galactolipids for the biogenesis of plastids and functioning of the photosynthetic machinery. In Arabidopsis, the first step of galactolipid synthesis is catalyzed by MGD1, a monotopic protein located in the inner envelope membrane of chloroplasts, which transfers a galactose residue from UDP-galactose to diacylglycerol (DAG). MGD1 needs anionic lipids such as phosphatidylglycerol (PG) to be active, but the mechanism by which PG activates MGD1 is still unknown. Recent studies shed light on the catalytic mechanism of MGD1 and on the possible PG binding site. Particularly, Pro189 was identified as a potential residue implied in PG binding, and His155 as the putative catalytic residue. In the present study, using a multifaceted approach (Langmuir membrane models, Atomic Force Microscopy, Molecular Dynamics), we investigated the membrane binding properties of native MGD1 and mutants (P189A and H115A). We demonstrated that both residues are involved in PG binding, thus suggesting the existence of a PG-His catalytic dyad that should facilitate deprotonation of the nucleophile hydroxyl group of DAG acceptor. Interestingly, molecular dynamics simulations showed that MGD1 induces a reorganization of lipids by attracting DAG molecules to create an optimal platform for binding.
\end{abstract}

Keywords: galactolipids, chloroplast, Arabidopsis, MGD1, reaction mechanism, phosphatidylglycerol, sulfoquinovosyldiacylglycerol, Langmuir monolayers, atomic force microscopy, molecular dynamics,

\title{
SIGNIFICANCE STATEMENT
}

Galactoglycerolipids are essential for the biogenesis of chloroplast membranes and functioning of photosynthetic machinery. In Arabidopsis, the chloroplastic membrane protein MGD1 is the major galactolipid synthase providing the bulk of monogalactosyldiacylglycerol needed for the massive expansion of thylakoids. This paper gives insight in the reaction mechanism and membrane binding properties of MGD1, highlighting the catalytic role of anionic lipids such as phosphatidylglycerol and the clustering of diacylglycerol acceptor substrate upon MGD1 binding. 


\section{INTRODUCTION}

A unique feature of chloroplast membranes is their high content of the uncharged galactolipids monogalactosyldiacylglycerol (MGDG) and digalactosyldiacylglycerol (DGDG), which represent up to $80 \%$ of the total lipid content (Block et al., 1983). Other lipids mostly consist of the anionic phosphatidylglycerol (PG) and sulfoquinovosyldiacylglycerol (SQDG). MGDG and DGDG play an important role in photosynthesis, particularly in stabilizing photosystems protein complexes at the level of thylakoid membranes (Jones, 2007; Kobayashi, 2016; Mizusawa and Wada, 2012). Galactolipids are also key elements in determining the membrane architecture (Demé et al., 2014; Gounaris and Barber, 1983; Gounaris et al., 1986). Indeed, the MGDG/DGDG ratio in chloroplasts seems crucial for the functional integrity of the photosynthetic machinery and therefore must be tightly regulated (Dörmann and Benning, 2002; Rocha et al., 2018).

Three functional MGDG synthases have been identified in Arabidopsis, denoted as MGD1, MGD2, and MGD3. They catalyze the transfer of a galactose residue from uridine 5'diphosphate- $\alpha$-D-galactose (UDP-Gal) to the sn-3 position of diacylglycerol (DAG) acceptor to form MGDG. MGD1 is the major isoform responsible for the synthesis of the bulk of MGDG ( $\sim 50$

$\%$ of total lipids) needed for the rapid and massive expansion of membrane thylakoids in response to light (Kobayashi et al., 2007). MGD2 and MGD3 are mostly produced in non-photosynthetic tissues and more specifically, they are induced in response to phosphate shortage (Awai et al., 2001; Kobayashi et al., 2009).

MGD1 is a monotopic membrane protein embedded in the outer leaflet of inner envelope membrane (IEM) (Miège et al., 1999; Vojta et al., 2007; Xu, 2005). The membrane binding properties of MGD1 are crucial for the enzyme function since it must bind to both the water-soluble donor sugar (UDP-Gal) and hydrophobic DAG acceptor substrates. The three-dimensional structure of MGD1, recently solved in its apo form and in complex with UDP, allows getting a more precise idea of its mode of action and its interaction with the membrane (Rocha et al., 2016). MGD1 adopts the classical GT-B fold consisting in two Rossmann-type domains separated by a large cleft forming the catalytic site. The C-terminal domain corresponds to the nucleotide-sugar binding domain, while the N-terminal domain is very likely involved in the binding of the DAG acceptor. Structural data provide insight into the residues of the active site and led us to propose 
the histidine 155 (H155) residue as the catalytic base that deprotonates the nucleophile OH group of DAG. One of the peculiarities, highlighted by the resolution of the structure, is the presence of a long and flexible region of nearly 50 amino acids in the N-terminal domain (not visible in the crystallographic structure) that is possibly involved in the capture of the DAG acceptor. Indeed, deletion of part of this region dramatically affected the rate of binding to DAG (Rocha et al., 2016). The current model proposes that MGD1 is inserted into the bilayer essentially by its N-terminal domain via this flexible region.

MGD1 needs anionic lipids such as phosphatidylglycerol (PG) or phosphatidic acid (PA) to be active (Dubots et al., 2010; Rocha et al., 2013). Intriguingly, PG and PA appear to proceed through different mechanisms and their effects are synergic, thus indicating distinct binding sites (Dubots et al., 2010). PA acts as an allosteric activator of MGD1 and is also a precursor for DAG but current data give no indication on its potential binding site in the protein. In contrast to PA, PG behaves as a classical activator of MGD1 (Dubots et al., 2010). Recently, it has been proposed that PG, which is abundant in plastid membranes (7-8 mol\%), contributes to the anchoring of MGD1 at the membrane surface in specific sites where DAG may transiently accumulate (Rocha et al., 2016). This assumption was substantiated by mutational studies indicating that PG binds to MGD1 in a region close to the DAG binding site. Specifically, point mutations of W287 and P189 to alanine resulted in loss of MGD1 activity in the presence of PG, whereas these mutants retain significant activity with PA (Dubots et al., 2010, Rocha et al., 2016).

The influence of lipid composition on MGD1 membrane binding was recently investigated using the Langmuir monolayer technique, which is particularly well adapted for monotopic membrane proteins (Sarkis et al., 2014). The Langmuir technique was also used to demonstrate the role of the flexible region in the N-domain in DAG binding (Rocha et al., 2016). MGD1 behaved differently when tested with pure MGDG or DGDG monolayers. MGDG, its reaction product, exerts a positive effect on MGD1 binding, whereas DGDG has a negative effect and tends to exclude the protein. These opposite effects suggest that MGD1 localizes to specific MGDG-enriched micro-domains but they also highlighted the importance of the MGDG/DGDG ratio in maintaining the enzyme bound to the membrane. Electrostatic interactions also contribute significantly to MGD1 binding as demonstrated in tests with monolayers made of either PA or PG (Sarkis et al., 2014). The effect was particularly pronounced with PG, which demonstrated high affinity for MGD1 and which was shown to counteract the negative effect of DGDG. 
To synthesize MGDG, MGD1 needs in addition to its donor sugar UDP-Gal to have access to its DAG acceptor, which is characterized by the absence of a polar head group. Taking into account that the amount of DAG in plastid membranes is very low (less than $1 \mathrm{~mol} \%$ ) and that the flux of DAG must be strictly regulated to meet the high demand in galactolipids, we hypothesized that MGD1 binds in specific sites where DAG may accumulate. We also considered that PG, which is abundant in the IEM ( $8 \mathrm{~mol} \%$ ), could help MGD1 to trap its DAG substrate (Rocha et al., 2016, 2018). This assumption was strengthened by the observation that MGD1 exhibited the fastest kinetics of adsorption on a DAG-PG $(1: 3 \mathrm{~mol} / \mathrm{mol})$ monolayer (Sarkis et al., 2014).

In the present study, we aimed to better understand the role of $\mathrm{PG}$, alone or in association with DAG, in MGD1 membrane binding. For this purpose, using a multifaceted approach combining biophysical (Langmuir experiments, Atomic Force Microscopy) and computational methods (Molecular Dynamics - Coarse Grain), we explored the binding properties of native MGD1 and two protein mutants (P189A and H155A) to various membrane models composed of pure PG or DAG or mixtures of both lipids at different molar ratios. A particular attention has been given to the residues involved in the interaction with the acceptor substrate (H155) on the one hand, and the PG phospholipid (P189) on the other hand, to give insights in the mechanism of activation of MGD1 by PG.

\section{RESULTS}

We aimed to better understand the interaction of MGD1 with lipid monolayers formed at the air/liquid interface with either pure DAG (acceptor substrate) or PG (activator), or mixtures of both lipids used at different molar ratios [DAG-PG (1:3 mol/mol) or DAG-PG $(3: 1 \mathrm{~mol} / \mathrm{mol})]$. The Langmuir monolayer technique was chosen for its simple experimental design and its suitability for studying the insertion of monotopic membrane proteins like MGD1 (Sarkis et al., 2014). In addition to native MGD1, two protein mutants (designated P189A and H155A) were included in the study. Proline 189 has been identified as a residue possibly interacting with PG and histidine 155 was proposed as the catalytic base for the transfer reaction (Dubots et al., 2010; Rocha et al., 
2016). These two residues were mutated into alanine, a substitution that is expected to preserve native protein structure and that does not introduce unwanted steric or electrostatic effects. The three proteins were purified to homogeneity using a two-step procedure (Rocha et al., 2013) and their enzyme activities were determined using the bioluminescent UDP-Glo ${ }^{\mathrm{TM}}$ Glycosyltransferase assay from Promega. This assay was found appropriate and very sensitive to monitor MGD1 activity and it can replace advantageously the classical radioactivity-based assay that was used so far (Rocha et al., 2013). We therefore checked that MGD1 showed no activity in the absence of an anionic lipid and greater activity in the presence of PA $(1230+/-90 \mathrm{nmol} / \mathrm{min} / \mathrm{mg}$ protein) compared to $\mathrm{PG}(482+/-21 \mathrm{nmol} / \mathrm{min} / \mathrm{mg}$ protein). We confirmed that the mutant H155A is totally inactive in the presence or absence of anionic lipids, and that the mutant P189A is inactive in the presence of PG and retained significant activity in the presence of PA (approximately $50 \%$ of the wild type enzyme). SQDG was also tested as a possible MGD1 activator since it was previously shown to greatly stimulate MGDG synthesis activity using a solubilized and partially purified enzyme extract from spinach chloroplast envelope membranes (Covés et al., 1988). In our tests, SQDG demonstrated the same capability to activate MGD1 as did PG and PA (891+/-30 $\mathrm{nmol} / \mathrm{min} / \mathrm{mg}$ protein). Interestingly, the P189A mutant was totally inactive in the presence of SQDG thus suggesting a similar binding site for PG and SQDG.

\section{Interfacial behavior of MGD1 and mutants at an air/buffer interface}

We characterized the surfactant properties of native MGD1 and mutants by evaluating their interfacial behavior at the air/buffer interface and without lipids. After injection of the protein under a nude interface, the adsorption of the protein at the air/buffer interface was monitored by continuous measurement of the surface pressure increase (П) as a function of time until the equilibrium value, $\Pi_{e}$, was reached. This surface pressure variation is directly proportional to the surfactant properties of the protein (Pitcher et al., 2002; Sarkis et al., 2011). The same $\Pi_{\mathrm{e}}$ value was obtained for the three proteins, around 14-15 mN/m (Figure 1a), indicating that the point mutations did not change significantly their surfactant properties and that these proteins behaved similarly at the air-liquid interface. 
Adsorption of MGD1 and mutants in an uncompressed monolayer of lipids: differential $\Pi_{0}$ $\left(d \Pi_{0}\right)$ parameter

It is possible to use the recently defined parameter, the differential $\Pi_{0}\left(\mathrm{~d} \Pi_{0}=\Delta \Pi_{0}-\Pi_{\mathrm{e}}\right)$, to evaluate the influence of the presence of lipids on the interfacial adsorption of the proteins at null surface pressure (uncompressed interface) (Nasir et al., 2017). A positive $\mathrm{d}_{0}$ value is indicative of an attractive effect of the lipids on the molecule adsorption onto the monolayer, whereas a negative $\mathrm{d} \Pi_{0}$ value indicates an unfavorable impact. The $\Delta \Pi_{0}$ values have been determined from plots of the maximum surface pressure increase $(\Delta \Pi)$ as a function of $\Pi_{i}$ using a DAG-PG $(1: 3 \mathrm{~mol} / \mathrm{mol})$ monolayer (Figure 1b). For MGD1 and mutants, the $\mathrm{d}_{0}$ values were positive, suggesting an attractive effect of the lipids on the interfacial adsorption of these different proteins with a significantly higher $\mathrm{d} \Pi_{0}$ value for the two mutants $\left(\mathrm{H} 155 \mathrm{~A} \mathrm{~d} \Pi_{0}=7.65 \pm 0.8 \mathrm{mN} / \mathrm{m}, \mathrm{P} 189 \mathrm{~A}\right.$ $\left.\mathrm{d} \Pi_{0}=8.91 \pm 0.6 \mathrm{mN} / \mathrm{m}\right)$ compared to native MGD1 ( $\left.\mathrm{d}_{0}=5.23 \pm 0.9 \mathrm{mN} / \mathrm{m}\right)$ (Figure 1c). Therefore, the uncompressed lipids attracted a little bit more mutants than the native enzyme. One plausible explanation is that mutations led to subtle conformational changes that either modified the amphiphilic balance of the protein which exposed differently hydrophobic/hydrophilic areas (i.e. H155A), or locally destructured the protein somewhat (i.e. P189A). Similar results were obtained with the other monolayers used in this study (Figure S1).

\section{Membrane binding properties of MGD1 and mutants on DAG and/or PG monolayers}

The plots of the maximum of surface pressure increase $(\Delta \Pi)$ measured upon injection of the protein at a fixed and subsaturating concentration under the monolayer, as a function of the initial pressure $\Pi_{\mathrm{i}}$ (from 7.5 to $25 \mathrm{mN} / \mathrm{m}$ ) allowed for determining two other important binding parameters: the maximum insertion pressure (MIP) and the synergy factor $a$ (slope +1 ) (Figure 1b). MIP corresponds to the surface pressure beyond which no adsorption can occur and provides information about the insertion ability of the molecule into a lipid membrane. If the MIP value is larger than the estimated membrane lateral pressure $(\sim 30 \mathrm{mN} / \mathrm{m})$ in natural biological membranes (Marsh, 1996), there is a significant interaction between the protein and the monolayer (Calvez et al., 2009). The synergy factor $a$ highlights the protein preference for a monolayer (Calvez et al., 2011). A positive $a$ value is indicative of a favorable interaction between the protein and 
monolayer, a negative value indicates repulsion, and a null synergy factor corresponds to a stationary state (with no favored or disfavored binding). Different monolayers were tested: pure DAG and PG monolayers and a DAG-PG mixture at 1:3 or 3:1 molar ratios. Figure S1 shows the plots obtained for MGD1 and mutants with the four different monolayers and Figure 2 summarizes the calculated MIP and $a$ values. The native enzyme did not show significant differences for the four monolayers tested, with a MIP value around 36 to $40 \mathrm{mN} / \mathrm{m}$ and a synergy factor in the range 0.4-0.5. The two mutants behaved almost similarly as native MGD1 on DAG-PG (3:1) and on DAG monolayers, although P189A exhibited significantly higher values (MIP $=48.7 \pm 3.6 \mathrm{mN} / \mathrm{m}$; $a=0.56 \pm 0.02$ ) compared to H155A mutant (MIP $=40.7 \pm 3.5 \mathrm{mN} / \mathrm{m} ; a=0.46 \pm 0.04)$. For the three proteins, the introduction of a limited amount of the negatively charged PG to the neutral DAG lipid appears to be slightly favorable to protein insertion. However, an excess of PG has an opposite effect and this is particularly true for the protein mutants. Marked differences were observed between mutants and native enzyme when tested on pure PG monolayer. The P189A mutant exhibited lower affinity towards PG as compared to MGD1. The MIP and $a$ values decreased to $30.9 \pm 0.09 \mathrm{mN} / \mathrm{m}$ and $0.2 \pm 0.02$, respectively, for the P189A mutant compared to MGD1 (MIP = $35.9 \pm 3.6 \mathrm{mN} / \mathrm{m} ; a=0.43 \pm 0.05)$. The most striking result came from the H155A mutant, which showed the lowest MIP and $a$ values for PG monolayer (MIP $=28.2 \pm 0.7 \mathrm{mN} / \mathrm{m}$ and $a=0.1 \pm 0.02$ ). Unexpectedly, these results suggest that $\mathrm{H} 155$ also interacts with PG. The results obtained with a PG-enriched DAG-PG (1:3) monolayer confirmed this assumption. The MIP values were roughly similar for the three proteins $(36.2 \pm 3.7 \mathrm{mN} / \mathrm{m}, 35.1 \pm 2.6 \mathrm{mN} / \mathrm{m}$ and $34.5 \pm 1.9 \mathrm{mN} / \mathrm{m}$ for MGD1, H155A and P189A, respectively). However, the $a$ values of mutants $(0.36 \pm 0.04$ and $0.33 \pm 0.03$ for H155A and P189A, respectively) were significantly smaller compared to MGD1 (0.44 \pm 0.05$)$ and, moreover, they were intermediate between those obtained with PG and with DAG-PG $(3: 1)$. Altogether, these results showed a clear altered binding for both mutants on a PG monolayer compared to the native enzyme, thus confirming the previously proposed role for P189 (Rocha et al., 2016) and revealing an unexpected interaction between H155 and PG.

\section{Organization of MGD1 and mutants on DAG and/or PG monolayers}


In order to complete our study on the binding parameters, AFM was used to image, at the mesoscopic scale, MGD1 and mutants inserted into DAG, DAG-PG (1:3), or PG monolayers. Experiments were done at initial surface pressure of $15 \mathrm{mN} / \mathrm{m}$ that was previously shown to be the most favorable $\Pi_{i}$ value for studying MGD1 binding (Sarkis et al., 2014).

In absence of protein, the monolayers transferred onto mica showed homogenous films with no visible phase separation (Figure 3, insets in panels 3a, 3d and 3g). Comparison of control AFM images with those obtained in the presence of native MGD1 revealed bright spots that are particularly visible and uniformly distributed on the DAG-PG (1:3) monolayer (Figure 3a). These bright spots can be attributed to protein or protein/lipid complexes embedded in a darker matrix corresponding to the lipid monolayer. In the case of DAG monolayer, the size of these clusters are even larger (Figure 3g). In contrast, MGD1 adsorbed homogenously on PG monolayer with no apparent lipid/protein cluster formation (Figure 3d). The P189A mutant behaves similarly as the native enzyme on PG (Figure 3e) and DAG-PG (Figure 3b) monolayers. However, its behavior is distinct from MGD1 on DAG monolayer (Figure 3h) in that images showed no bright patches, but instead, homogenous distribution as observed on PG. Images obtained with H155A mutant presented a few differences. Bright spots were evidenced for the three types of monolayers, more noticeably on DAG-PG. However, this mutant seemed to induce a reorganization of the monolayer (visible as dark spots on Figure $3 \mathrm{c}$ and $3 \mathrm{f}$ ) that was not observed with the P189A mutant nor with MGD1. This effect is not seen on DAG monolayer (Figure 3i).

\section{Interaction of MGD1 with PG and DAG by computer modeling}

Using Molecular Dynamics simulation, in the Coarse-Grain approximation, we investigated the interactions of MGD1 with a DAG-PG bilayer (at molar ratio 1:3) at the nanoscale level (Figure 4a). During the whole trajectory, the protein remained tightly bound to the bilayer surface (but it undergoes internal motions of its $\mathrm{N}$ - and $\mathrm{C}$-domains vide supra). The most striking feature is the observation that the overall set of interactions between MGD1 and the lipids induces a significant clustering of DAG molecules. Without protein, DAG and PG are well mixed, as indicated by the number of contacts between these lipids in the membrane : $80 \%$ of DAG molecules interact with PG molecules along with $20 \%$ of DAG molecules are involved in self-contacts and $33 \%$ of PG molecules interact with DAG molecules while $67 \%$ of PG molecules form self-contacts. This is in 
agreement with the overall content of PG and DAG (75\% and $25 \%$, respectively) in the model membrane. The presence of MGD1 induces a reorganization of the lipids that results in a higher local concentration of DAG molecules, which assembles in the form of clusters. Such a clustering effect is clearly illustrated by the number of contacts of MGD1 with DAG and PG. On average, there are $31 \pm 8$ DAG molecules interacting with MGD1, whereas this number amounts to $12 \pm 4$ for PG molecules. A snapshot from the trajectory given in Figure $4 \mathrm{~b}$ illustrates the location of PG and DAG molecules with respect to the protein.

During the course of the simulation, we observed intramolecular motions within MGD1 involving the two Rossmann-type domains corresponding, respectively, to the $\mathrm{N}$ - and C-moieties of the protein. While the $\mathrm{N}$-domain always remains anchored in the membrane, the $\mathrm{C}$-domain experiments excursions that brings it in the vicinity of the membrane (Figure 4c). At this position, the interactions between both surface groups of $\mathrm{C}$-domain and residues of the catalytic site with PG molecules become possible. Then the protein returns to the initial position. The exchange between the two orientations of the protein occurs within $1 \mu \mathrm{s}$. This observation pertains to the dynamics of the relative positions of the two key residues P189 and H155 and their interactions with PG. P189 is located in the N-domain on the long and flexible modeled loop, which is buried into the membrane. The catalytic residue $\mathrm{H} 155$ also belongs to the $\mathrm{N}$-domain and it is located in the large cleft which hosts the active site (Figure 4a). The distance between these two residues is $20 \AA$ and it remains constant during the trajectory. In the initial state, H155 is remote from the membrane surface and there are no lipid molecules within a $8 \AA$ distance (this corresponds to the first solvation shell in the Martini model and was used as a cut-off). Therefore, the interactions of P189 with PG molecules are possible due to the location of this residue within the membrane while the constraint for H155 to interact with PG requires the approach of C-domain to the membrane surface. The oscillations between the orientations of the $\mathrm{C}$-domain with respect to the membrane surface would explain part of the reaction mechanism. Besides entrapping PG and DAG, MGD1 needs to entrap the UDP-Gal donor and then release UDP. The change in protein orientation with respect to the membrane could provide the switch between two processes, i.e. binding of hydrophilic donor substrate and of hydrophobic acceptor substrate.

\section{DISCUSSION}


In Arabidopsis, MGD1 is a key enzyme in the biogenesis of chloroplast membranes to provide the bulk of galactolipids needed for the massive expansion of thylakoids. This MGDG synthase has been extensively studied at the physiological, biochemical and structural levels (for a review see, (Rocha et al., 2018)). MGD1 is a monotopic membrane protein embedded in the IEM, which requires anionic lipids such as PG or PA to be active (Dubots et al., 2010). Kinetics data indicated that PG and PA proceed through different mechanisms and probably have distinct binding sites. The recent 3D structure of MGD1 shed light on the catalytic mechanism and highlighted the role of $\mathrm{H} 155$ as the catalytic base. It also pointed out the importance of a large and flexible loop in DAG binding (Rocha et al., 2016). However, structural data did not reveal the role of anionic lipids in catalysis. Previous studies, based on the use of Langmuir membrane model, showed that the lipid composition has an influence on the membrane binding properties of MGD1 (Sarkis et al., 2014). MGD1 demonstrated high affinity for its DAG substrate, as expected, but also for PG and PA. Particularly, MGD1 showed the highest rate of binding for a DAG-PG lipid combination. The role of PA in MGD1 regulation is of paramount importance in a physiological context since it is an allosteric activator of MGD1 and also a central metabolite in the glycerolipid metabolism pathway (Dubots et al., 2011). Unfortunately, adding PA to a DAG monolayer generated instability that makes results difficult to interpret. We therefore focused our study on the interplay between PG, DAG and MGD1 with the aim to unravel the role of PG as MGD1 activator. We took advantage of the identification of a particular amino acid residue in MGD1, P189, possibly involved in PG binding (Dubots et al., 2010; Rocha et al., 2016) to explore its impact on the interaction of MGD1 on various membrane models. In addition, this proline residue is located in the large and flexible region that was proposed to be essential to trap the DAG acceptor (Rocha et al., 2016).

In the present study, we investigated the influence of the lipid composition on the ability of MGD1 and of two protein mutants, P189A and H155A, to interact with a membrane. To this end, we used Langmuir monolayers as biomimetic membrane models, composed of either DAG or PG or a combination of both lipids, to better understand the effects of the one-point mutation on the membrane anchoring process. The mutation of these two amino acids into alanine, which was expected not to alter significantly the protein conformation, did not change the surfactant properties of mutant proteins determined at the air/buffer interface (Figure 1a). However, the presence of the lipid acyl chains (i.e. hydrophobic environment), at null surface pressure (uncompressed interface), 
exerted a slightly better attractive effect on the interfacial protein adsorption of mutants, as shown by the determination of the differential parameter $\mathrm{d} \Pi_{0}$ (Figure 1c). This suggests that the protein amphiphilic balance was modified by subtle conformational changes induced by mutations, and therefore, any decrease in the values of the binding parameters (MIP and synergy factor $a$ ) can be interpreted as a loss of the interaction capabilities of the mutants with the membrane.

The native MGD1 showed a similar behavior with the four monolayers tested (Figure 2) with MIP values ranging from 36 to $40 \mathrm{mN} / \mathrm{m}$ and synergy factors from 0.43 to 0.51 , confirming its high affinity for these lipid species (Sarkis et al., 2014). The P189A mutant behaved similarly as the native enzyme on DAG and DAG-PG (3:1). However, the affinity of this mutant decreased significantly when tested on pure PG, with a 2-fold decrease of the synergy factor. Interestingly, an intermediate value was obtained when using a DAG-PG with inverted molar ratio (1:3). These results reinforce the hypothesis that the P189 residue is involved in PG binding. The H155A mutant was included in the study to corroborate its role as the catalytic base. This mutant displays no catalytic activity, which is consistent with its proposed role (Rocha et al., 2016). Therefore, it was interesting to see if this mutation also affects the membrane binding properties. This mutant displayed a similar behaviour as the native enzyme when tested on DAG and DAG-PG (1:3) monolayers. Unexpectedly, the affinity of H155A mutant towards PG dropped drastically with a synergy factor close to 0 , suggesting a stationary state without any favoured or disfavoured interaction for protein binding. Adding DAG to the PG monolayer (1:3 molar ratio) restored affinity to some extent. These results suggest that His 155 effectively interacts with PG and that this electrostatic interaction is a major contributor to MGD1 binding onto PG membrane.

Although MGD1 demonstrated similar binding parameters for the various monolayers, the native enzyme organized differently at the membrane interface depending on the lipid nature (Figure 3). Homogenous binding was observed in the presence of PG with no visible protein clusters on AFM images. By contrast, the presence of DAG favoured protein-protein interactions, and the formed complexes were particularly large on pure DAG monolayer. The P189A mutant has a similar high affinity for DAG binding (as seen in Figure 2) but it lost its capacity to form large protein aggregates. This proline residue is located in the N-domain, in a region that was previously proposed to interact with the membrane (Rocha et al., 2016). Point mutation of this residue presumably changed locally the conformation of the polypeptide chain, which became less constrained, and incidentally, impacted its propensity to form protein clusters. The mutation of 
H155 into alanine was not expected to alter significantly protein organization. However, on DAG monolayer, H155A mutant also lost its capacity to form large aggregates. Intriguingly, AFM images showed that this mutant induced a reorganization of the monolayers in the presence of PG, with the formation of holes suggesting a phenomenon of lipid segregation.

Extension of the results derived from lipid monolayers could be achieved throughout the extensive molecular modeling simulation performed on a bilayer membrane, which may have more biological relevance. Over the course of the MD simulation we did not observe any membrane curvature, thus allowing a direct comparison of results of the simulation with experimental data obtained on a flat lipid monolayer. Upon the course of the simulation, we observed an increase of the local concentration of DAG molecules in the vicinity of the protein, which is the result of the interactions between residues at the protein surface and the glycerol moiety of DAG. This provides an explanation of the well-distributed protein patches observed on DAG-PG (1:3) monolayer (Figure 3a). This also suggests an ordering of membrane lipids in vivo with DAG clustered by MGD1. It will be of interest to investigate whether other local membrane reorganization might occur with other lipids, in particular PA, and how this affects other DAG-using enzymes such as SQDG synthase.

The molecular dynamics simulation offers an extension of the motions that happen in the proteins and its surrounding over a time scale in line with those generally observed for such reaction mechanism. In particular, we observed spatial oscillations of the C-domain of MGD1 with respect to the membranes which is accompanied by a concerted motion that brings the H155 residue of active site close to membrane surface for the recruitment of PG. Therefore, the interactions of P189 with PG molecules are possible due to the expected location of this residue within the membrane. As such, we provide a structural information explaining the key role played by H155 and P189 in binding PG. But besides entrapping PG and DAG, MGD1 needs to entrap the UDP-Gal donor and then release UDP. The change in protein orientation with respect to the membrane could provide the switch between two processes, i.e. binding of hydrophilic donor substrate and of hydrophobic acceptor substrate.

Based on the present data, a reaction mechanism for MGD1 is proposed that gives insight into the role of the enzyme activator PG. Enzyme reaction is suggested to occur in a single displacement $\mathrm{S}_{\mathrm{N}} 2$ mechanism, with formation of an oxocarbenium-ion transition state assisted by 
a catalytic base (H155) (Rocha et al., 2016). In this model, PG binds to the active site where it is stabilized by interactions involving P189 and H155 (Figure 5). This creates an acid-base charge relay system, in the form of a PG-His dyad that should facilitate deprotonation by $\mathrm{H} 155$ of the OH group of DAG acceptor. Such acid-base dyads (involving a Glu or Asp instead of PG) have been described in some bacterial glycosyltransferases (for a review, see (Breton et al., 2012). This model also implies that a DAG molecule co-localizes with a PG molecule, thus strengthening the hypothesis that the large flexible region in the $\mathrm{N}$-domain contributes to both the anchoring of MGD1 to the membrane and to the capture of PG and DAG. At the time of writing, we found no example in the literature of a similar catalytic dyad involving a negatively charged lipid. Other anionic lipids can fulfil the same role as PG. SQDG, which is another important component of chloroplast membranes ( $\sim 10 \%$ of total lipids), can also activate plant MGDG synthases ((Covés et al., 1988), this study). Kinetic analysis of P189A demonstrated that the mutant is inactive in the presence of SQDG, suggesting that the sulfolipid binds to the same site as PG. Our model raises the issue of the mechanism of activation of MGD1 by PA since it is expected to bind to a distinct site (Dubots et al., 2010). Our hypothesis is that PA, which has a small polar head compared to PG and SQDG, can bind to the active site but in a different way, making no direct interaction with P189 but maintaining hydrogen bonding to His155. One cannot exclude the possibility of a second binding site for PA, which would be a regulator site, thus explaining the allosteric behaviour of PA.

PG is described as an essential component in structures and functions of photosystems (for a recent review, see (Kobayashi et al., 2017)). In addition, PG and SQDG appear to be functionally redundant in plant chloroplasts (Yu et al., 2002) and their respective levels are adjusted to maintain total anionic lipid content $(\sim 15 \%)$. We herein demonstrated the importance of PG in membrane binding and reaction mechanism of MGD1, which is a central enzyme in the biogenesis of chloroplast membranes (Rocha et al., 2018). It remains to be determined, in a physiological context, which anionic lipid (SQDG or PG) will be the preferred MGD1 activator.

\section{MATERIALS AND METHODS}

\section{Materials}


DAG (1-2-dioleoyl-sn-glycerol) was purchased from Avanti Polar Lipids (Alabama, US) and PG (L- $\alpha$-Phosphatidyl-DL-glycerol ammonium salt from egg yolk lecithin) from Sigma-Aldrich (Saint-Quentin Fallavier, France). UDP-Gal was from Promega (Charbonnières-les-Bains, France).

\section{Lipid preparation}

The solvents were of analytical grade (Sigma-Aldrich, Saint-Quentin Fallavier, France). The lipid mixtures were prepared at a concentration of $1 \mathrm{mM}$ in chloroform and stored at $-20^{\circ} \mathrm{C}$ under argon to prevent lipid oxidation.

\section{Expression and purification of MGD1}

The generation of the MGD1 catalytic domain protein construct comprising residues [137-533] fused to a His6-tag at the C-terminus has been described previously (Rocha et al., 2013). Briefly, this sequence was cloned into pET29b vector (Novagen Inc.) and the resulting plasmid was used to transform E. coli BL21(DE3) cells. Conditions of expression and purification of MGD1were as previously described (Rocha et al., 2013). The pure protein $(10 \mathrm{mg} / \mathrm{ml})$ in Bis-Tris buffer $[25 \mathrm{mM}$ Bis-Tris, $150 \mathrm{mM} \mathrm{NaCl}, 1 \mathrm{mM}$ Tris(2-carboxyethyl) phosphine hydrochloride (TCEP), 5\% Glycerol; pH 6.5] was stored at $-20^{\circ} \mathrm{C}$ until use. Protein mutants H155A and P189A have been generated from the same plasmid using the QuickChange Site-directed mutagenesis kit (Agilent Technologies, France). Mutant proteins were expressed and purified as the wild-type.

\section{MGD1 activity assay}

Enzyme activity was assayed in mixed micelles using a protocol adapted from (Rocha et al., 2013). Enzyme assays were performed at room temperature in Bis-Tris buffer supplemented with $6 \mathrm{mM}$ CHAPS, in the presence of either $1.5 \mathrm{~mol} \%$ PG or PA, $6.3 \mathrm{~mol} \% \mathrm{DAG}$, and $1 \mathrm{ng}$ (PA tests) or 5 ng (PG tests) of enzyme in a final volume of $36.5 \mu 1$. The micellar surface concentration of lipids (expressed in mol\%) relative to all mixed micelle components was used to express the lipid concentration. Reaction is initiated by addition of UDP-Gal $(700 \mu \mathrm{M})$ and the release of UDP as a function of time is measured using the UDP-Glo ${ }^{\mathrm{TM}}$ Glycosyltransferase Assay from Promega. The 
principle of the assay is to convert UDP to ATP to generate light in a luciferase reaction. Twentyfive $\mu 1$ of reaction mix are mixed with $25 \mu 1$ of UDP Detection reagent in a 96-well microplate and after $1 \mathrm{hr}$-incubation at room temperature, the luminescence was read in a plate-reading luminometer (Spark 10M, Tecan). In our hands, the bioluminescent signal is proportional to UDP concentration in the range [0.01-0.8 $\mu \mathrm{M}]$. Assays were done in triplicates and enzyme activity was expressed in $\mathrm{nmol} / \mathrm{mn} / \mathrm{mg}$ protein. Control reactions were performed in the absence of acceptor.

\section{Langmuir experiments}

Monolayers were prepared on a KSV 2000 Langmuir-Blodgett trough (three multi-compartments, KSV NIMA, Biolin Scientific, Finland) with a symmetric compression system. The rectangular trough has a volume of $80 \mathrm{~mL}$ and a surface of $119.25 \mathrm{~cm}^{2}$. A Wilhelmy plate was used to measure the surface pressure $(\pi)$ with an accuracy of $\pm 0.5 \mathrm{mN} / \mathrm{m}$. The trough was cleaned with ethanol, dichloromethane and ultrapure water successively, and filled with filtered Bis-Tris buffer. In our previous study (Sarkis et al., 2014), buffer was composed of $50 \mathrm{mM} \mathrm{MOPS,} 23 \mathrm{mM} \mathrm{K}_{2} \mathrm{HPO}_{4}, 23$ $\mathrm{mM}$ KCL, $100 \mathrm{mM} \mathrm{NaCl}, 1 \mathrm{mM}$ DTT, 2\% glycerol $\mathrm{pH}$ 7.8, but this buffer was changed in the present study to be in the same composition as the protein buffer. The Bis-Tris buffer ( $\mathrm{pH}$ 6.5) was found more suitable for the activity and stability of MGD (Rocha et al., 2013). In addition, no significant differences were observed when MGD1 was tested on a PG monolayer with both buffers (data not shown). All experiments were performed at $25^{\circ} \mathrm{C}$ by maintaining the desired temperature with an Ecoline RE106 low-temperature thermostat (LAUDA, Germany).

The method of monolayer formation consisted of a drop of $15 \mu \mathrm{L}$ of the lipid mixture (1 $\mathrm{mM}$ in chloroform) gently spread at the air/buffer interface with a micro-syringe. After 15 minutes allowing solvent evaporation, the monolayer was compressed at different initial surface pressures $\Pi_{\mathrm{i}}(7.5,10,15,20,23,25 \mathrm{mN} / \mathrm{m})$ by two mobile barriers. After stabilization of the monolayer, the surface area was kept constant by stopping the movement of the mobile barriers and then, the protein was injected under the surface at a fixed and sub-saturating concentration $(13 \mathrm{nM})$. This injection triggered the increase of the surface pressure to attain the equilibrium surface pressure, indicating the end of the adsorption process. The changes in surface pressure $(\Delta \Pi)$ induced by the interaction of proteins with the monolayer were recorded continuously, as a function of time, with a computer-controlled Langmuir film balance KSV NIMA (Biolin Scientific, Finland). Each 
experiment was repeated at least three times for each set of conditions. All the lipid monolayers were in a fluid phase.

\section{Determination of the binding parameters of MGD1}

The parameters that characterize the binding of MGD1 to different membranes were assessed as previously described (Sarkis et al., 2014). The plot of the maximal surface pressure increase $\left(\Delta \Pi_{\max }\right)$ as a function of $\Pi_{i}$ allows the determination of the maximum insertion pressure (MIP) and the $\Delta \Pi_{0}$ values by extrapolating the regression of the plot to $x$ and $y$ axes, respectively. The synergy factor (a) was obtained by adding 1 to the slope of the plot (Boisselier et al., 2012). The uncertainties on MIP and the synergy factor $a$, were determined as previously described (Boisselier et al., 2012; Calvez et al., 2011). The uncertainty on MIP and $\Delta \Pi_{0}$ was calculated with a $95 \%$ confidence interval from the covariance of the experimental data for the linear regression. These experimental errors were directly determined with the free binding parameters calculator software (http://www.crchudequebec.ulaval.ca/BindingParametersCalculator) developed by Salesse's group. The differential $\Pi_{0}\left(\mathrm{~d} \Pi_{0}\right)$ parameter was calculated as follows: $\mathrm{d} \Pi_{0}=\Delta \Pi_{0}-\Pi_{\mathrm{e}}$, where the $\Delta \Pi_{0}$ value reflects the insertion abilities of the protein in an uncompressed monolayer of lipids, and $\Pi_{\mathrm{e}}$ is the surface pressure increase at the equilibrium obtained with the protein in absence of lipids (Nasir et al., 2017).

\section{Atomic Force Microscopy (AFM)}

The same KSV 2000 Langmuir-Blodgett trough (three multi-compartment system, KSV NIMA, Biolin Scientific, Finland) with a symmetrical compression system was used to measure the surface pressure and prepare AFM samples by the way of Langmuir-Blodgett (LB) technique. After stabilization of the surface pressure, the Langmuir film was transferred to freshly cleaved mica plates by vertically raising $(1 \mathrm{~mm} / \mathrm{min})$ the mica through the lipid/liquid interface maintained at constant surface pressure of $15 \mathrm{mN} / \mathrm{m}$. AFM images of LB films were obtained in tapping mode using a Solver PRO atomic force microscope (NT-MDT Spectrum Instruments), under ambient conditions with a scanning area of $5 \times 5 \mu \mathrm{m}^{2}$. Topographic images were acquired using silicon nitride tips on integral cantilevers with a nominal spring constant of 1.45-15.1 N/m and a frequency 
between $87-230 \mathrm{kHz}$. Representative images were obtained from at least 2 samples prepared on different days and from $\geq 3$ macroscopically separated areas on each sample.

\section{Molecular dynamics simulations}

The initial structure of DAG-PG bilayer was built using the CHARMM-GUI online service (Qi et al., 2015) in the proportion 1:3. In the initial system, both leaflets of the bilayer were symmetric. The corresponding membrane in water box without protein was calculated as a reference, the box of $21.5 \times 21.5 \times 7.3 \mathrm{~nm}$ size contained 1200 PG and 400 DAG molecules, 11988 water molecules and $1349 \mathrm{Na}+$ and $149 \mathrm{Cl}$ - atoms. The 3-dimensional structure of MGD1 was taken from the reported X-ray solved structure (PDB code: 4WYI) (Rocha et al., 2016). The long and flexible region in the $\mathrm{N}$-domain of MGD1, not visible in the crystal structure, was constructed de novo using online service i-Tasser (Roy et al., 2010). In the initial system, both leaflets of the bilayer were symmetric except for the elimination of several lipid molecules to compensate the partial protein burying into the membrane. The initial orientation of the protein to the bilayer was set using the OPM service which minimizes the energy for transferring the hydrated protein to the membrane (Lomize et al., 2012). The composition of the macromolecular system was: 1190 PG molecules and 393 DAG molecules, one MGDG1 protein, 28151 water molecules. The electrostatic neutralization required the addition of $1502 \mathrm{Na}^{+}$and $324 \mathrm{Cl}^{-}$atoms. An overall salt concentration of approximately $150 \mathrm{mM}$ (excluding counter ions) was obtained. The initial size of the macromolecular system was $21.5 \times 21.5$ in the lateral $(\mathrm{x}, \mathrm{y})$ dimensions and $11.8 \mathrm{~nm}$ in the $\mathrm{z}$ dimension at the start of the simulation.

The molecular dynamics (MD) calculations were performed at the Coarse-Grain level of approximation with Martini 2.2 parameters (Marrink et al., 2007) using GROMACS 5.1 (Van Der Spoel et al., 2005). The systems were energy-minimized with the steepest descent algorithm within 10000 steps. Then followed five periods of equilibration (each for $1 \mu \mathrm{s}$ ) which were performed using a leapfrog integrator using appropriate time steps (ranging from 2 to $20 \mathrm{fs}$ ) and gradual decrease on restraints of the position of the lipid head groups. The production phase was carried out without any restraints, using time step of $20 \mathrm{fs}$. The temperature of the macromolecular system was kept at $298 \mathrm{~K}$ by a velocity rescale coupling algorithm with $2.0 \mathrm{ps}$ time constant. The pressure was kept at 1 bar using Berendsen barostat with 2.0 ps coupling constant and a compressibility of $3 \times 10^{-4}$ bar. The total length of the trajectory was $6 \mu \mathrm{s}$. For the analysis of averaged properties of 
the equilibrated membrane the last $4 \mu$ s were considered. The post-processing analysis was performed using the analytical tools implemented in GROMACS.

\section{Acknowledgments}

This work was supported by the French National Research Agency (ANR-10-Blan-1524 ReGal, ANR-13-BSV8-0011); Université Grenoble Alpes (AGIR 2015); and Communauté Université Grenoble Alpes (Cross-Disciplinary project Glyco@Alps, ANR-15-IDEX-02). We thank Dr Véronique Vié for helpful discussions.

Competing interests: The authors declare no conflict of interest

\section{Supporting information}

Figure S1: Increase of surface pressure $(\Delta \Pi)$ at equilibrium as a function of $\Pi$ i when native MGD1 and mutants interact with various lipid monolayers. Each point is the mean of two or three independent series of experiments.

Figure S2. Statistical analyses of MIP and $a$ values. 


\section{References}

Awai, K., Maréchal, E., Block, M.A., Brun, D., Masuda, T., Shimada, H., Takamiya, K., Ohta, H. and Joyard, J. (2001) Two types of MGDG synthase genes, found widely in both 16:3 and 18:3 plants, differentially mediate galactolipid syntheses in photosynthetic and nonphotosynthetic tissues in Arabidopsis thaliana. Proc. Natl. Acad. Sci. USA, 98, 10960-10965.

Block, M.A., Dorne, A-J., Joyard, J. and Douce, R. (1983) Preparation and Characterization of Membrane Fractions Enriched in Outer and Inner Envelope Membranes from Spinach Chloroplasts. J. Biol. Chem. 258, 13281-13286.

Boisselier, E., Calvez, P., Demers, E., Cantin, L. and Salesse, C. (2012) Influence of the Physical State of Phospholipid Monolayers on Protein Binding. Langmuir, 28, 9680-9688.

Breton, C., Fournel-Gigleux, S. and Palcic, M.M. (2012) Recent structures, evolution and mechanisms of glycosyltransferases. Curr. Opin. Struct. Biol. 22, 540-549.

Calvez, P., Bussières, S., Demers, E. and Salesse, C. (2009) Parameters modulating the maximum insertion pressure of proteins and peptides in lipid monolayers. Biochimie, 91, 718-733.

Calvez, P., Demers, E., Boisselier, E. and Salesse, C. (2011) Analysis of the Contribution of Saturated and Polyunsaturated Phospholipid Monolayers to the Binding of Proteins. Langmuir, 27, 1373-1379.

Covés, J., Joyard, J. and Douce, R. (1988) Lipid requirement and kinetic studies of solubilized UDP-galactose:diacylglycerol galactosyltransferase activity from spinach chloroplast envelope membranes. Proc. Natl. Acad. Sci. USA, 85, 4966-4970.

Demé, B., Cataye, C., Block, M.A., Maréchal, E. and Jouhet, J. (2014) Contribution of galactoglycerolipids to the 3-dimensional architecture of thylakoids. FASEB J. 28, 3373-3383.

Dörmann, P. and Benning, C. (2002) Galactolipids rule in seed plants. Trends Plant Sci. 7, 112-118.

Dubots, E., Audry, M., Yamaryo, Y., Bastien, O., Ohta, H., Breton, C., Maréchal, E. and Block, M.A. (2010) Activation of the Chloroplast Monogalactosyldiacylglycerol Synthase MGD1 by Phosphatidic Acid and Phosphatidylglycerol. J. Biol. Chem. 285, 6003-6011.

Dubots, E., Botté, C., Boudière, L., Yamaryo-Botté, Y., Jouhet, J., Maréchal, E. and Block, M.A. (2011) Role of phosphatidic acid in plant galactolipid synthesis. Biochimie, 94, 86-93.

Gounaris, K. and Barber, J. (1983) Monogalactosyldiacylglycerol: the most abundant polar lipid in Nature. Trends Biochem. Sci. 8, 378-381. 
Gounaris, K., Barber, J. and Harwood, J.L. (1986) The thylakoid membranes of higher plant chloroplasts. Biochem. J. 237, 313-326.

Jones, M.R. (2007) Lipids in photosynthetic reaction centres: Structural roles and functional holes. Prog. Lipid Res. 46, 56-87.

Kobayashi, K. (2016) Role of membrane glycerolipids in photosynthesis, thylakoid biogenesis and chloroplast development. J. Plant Res. 129, 565-580.

Kobayashi, K., Awai, K., Nakamura, M., Nagatani, A., Masuda, T., and Ohta, H. (2009) TypeB monogalactosyldiacylglycerol synthases are involved in phosphate starvation-induced lipid remodeling, and are crucial for low-phosphate adaptation. Plant J. 57, 322-331.

Kobayashi, K., Endo, K. and Wada, H. (2017) Specific Distribution of Phosphatidylglycerol to Photosystem Complexes in the Thylakoid Membrane. Front. Plant Sci. 8, 1-7.

Kobayashi, K., Kondo, M., Fukuda, H., Nishimura, M. and Ohta, H. (2007) Galactolipid synthesis in chloroplast inner envelope is essential for proper thylakoid biogenesis, photosynthesis, and embryogenesis. Proc. Natl. Acad. Sci. U.S.A 104, 17216-17221.

Lomize, M.A., Pogozheva, I.D., Joo, H., Mosberg, H.I. and Lomize, A.L. (2012) OPM database and PPM web server: resources for positioning of proteins in membranes. Nucleic Acids Res. 40, 370-376.

Marrink, S.J., Risselada, H.J., Yefimov, S., Tieleman, D.P. and De Vries, A.H. (2007) The MARTINI force field: Coarse Grained Model for Biomolecular Simulations. J. Phys. Chem. $B, 111,7812-7824$.

Marsh, D. (1996) Lateral pressure in membranes. Biochim. Biophys. Acta, 1286, 183-223.

Miège, C., Maréchal, E., Shimojima, M., Awai, K., Block, M.A., Ohta, H., Takamiya, K., Douce, R. and Joyard, J. (1999) Biochemical and topological properties of type A MGDG synthase, a spinach chloroplast envelope enzyme catalyzing the synthesis of both prokaryotic and eukaryotic MGDG. Eur. J. Biochem. 265, 990-1001.

Mizusawa, N. and Wada, H. (2012) The role of lipids in photosystem II. Biochim. Biophys. Acta, 1817,194-208.

Nasir, M.N., Lins, L., Crowet, J.M., Ongena, M., Dorey, S., Dhondt-Cordelier, S., Clément, C., Bouquillon, S., Haudrechy, A., Sarazin, C., Fauconnier, M-L., Nott, K. and Deleu, M. (2017) Differential Interaction of Synthetic Glycolipids with Biomimetic Plasma Membrane Lipids Correlates with the Plant Biological Response. Langmuir, 33, 9979-9987.

Pitcher, W. H., Keller, S. L. and Huestis, W.H. (2002) Interaction of nominally soluble proteins with phospholipid monolayers at the air-water interface. Biochim. Biophys. Acta, 1564, 107-113.

Qi, Y., Ingólfsson, H.I., Cheng, X., Lee, J., Marrink, S.J. and Im, W. (2015) CHARMM-GUI Martini Maker for Coarse-Grained Simulations with the Martini Force Field. J. Chem. 
Theory Comput. 11, 4486-4494.

Rocha, J., Audry, M., Pesce, G., Chazalet, V., Block, M.A., Maréchal, E. and Breton, C. (2013) Revisiting the expression and purification of MGD1, the major galactolipid synthase in Arabidopsis to establish a novel standard for biochemical and structural studies. Biochimie, 95, 700-708.

Rocha, J., Nitenberg, M., Girard-Egrot, A., Jouhet, J., Maréchal, E., Block, M.A. and Breton, C. (2018) Do Galactolipid Synthases Play a Key Role in the Biogenesis of Chloroplast Membranes of Higher Plants? Front. Plant Sci. 9, 1-7.

Rocha, J., Sarkis, J., Thomas, A., Pitou, L., Radzimanowski, J., Audry, M., Chazalet, V., de Sanctis, D., Palcic, M.M., Block, M.A., Girard-Egrot, A., Maréchal, E. and Breton, C. (2016) Structural insights and membrane binding properties of MGD1, the major galactolipid synthase in plants. Plant J. 85, 622-633.

Roy, A., Kucukural, A. and Zhang, Y. (2010) I-TASSER: a unified platform for automated protein structure and function prediction. Nat. Protoc. 5, 725-738.

Sarkis, J., Hubert, J.F., Legrand, B., Robert, E., Chéron, A., Jardin, J., Hitti, E., Le Rumeur, E. and Vie, V. (2011) Spectrin-like Repeats 11-15 of Human Dystrophin Show Adaptations to a Lipidic Environment. J. Biol. Chem. 286, 30481-30491.

Sarkis, J., Rocha, J., Maniti, O., Jouhet, J., Vie, V., Block, M.A., Breton, C., Marechal, E. and Girard-Egrot, A. (2014) The influence of lipids on MGD1 membrane binding highlights novel mechanisms for galactolipid biosynthesis regulation in chloroplasts. FASEB J. 28, 3114-3123.

Van Der Spoel, D., Lindahl, E., Hess, B., Groenhof, G., Mark, A.E. and Berendsen, H.J.C. (2005) GROMACS: Fast, flexible, and Free. J. Comput. Chem. 26, 1701-1718.

Vojta, L., Soll, J. and Bölter, B. (2007) Protein transport in chloroplasts - targeting to the intermembrane space. FEBS J. 274, 5043-5054.

Xu, C., Fan, J, Froehlich, J.E., Awai, K. and Benning, C. (2005) Mutation of the TGD1 Chloroplast Envelope Protein Affects Phosphatidate Metabolism in Arabidopsis. Plant Cell, 17, 3094-3110.

Yu, B., Xu, C. and Benning, C. (2002) Arabidopsis disrupted in SQD2 gene encoding sulfolipid synthase is impaired in phosphate-limited growth. Proc. Natl. Acad. Sci. U.S.A 99, 57325737. 


\section{FIGURE LEGENDS}

Figure 1: Adsorption of native MGD1, P189A and H155A mutants at the air/liquid interface in the absence and in the presence of lipids.

(a) Kinetics of adsorption of MGD1 (green), P189A (blue) and H155A (orange) mutants (each at $13 \mathrm{nM}$ ) at a nude air/liquid interface, after injection of proteins into the subphase at $25^{\circ} \mathrm{C}$.

(b) Adsorption of the native protein MGD1, P189A and H155A mutants to a DAG-PG (1:3 $\mathrm{mol} / \mathrm{mol})$ monolayer. Variation of surface pressure $(\Delta \Pi$ in $\mathrm{mN} / \mathrm{m})$ when proteins interacted with the monolayer at different initial surface pressure $\left(\Pi_{i}\right)$. This representation allowed determining MIP, $\Delta \Pi_{0}$, and synergy factor ( $a$ ) values.

(c) Differential $\mathrm{d} \Pi_{0}$ for the native and mutant proteins, calculated as follows: $\mathrm{d} \Pi_{0}=\Delta \Pi_{0}-\Pi_{\mathrm{e}}\left(\Pi_{\mathrm{e}}\right.$ being the plateau value determined in panel A).

Figure 2: Influence of the lipid nature on protein interactions. MIPs values $(\mathrm{mN} / \mathrm{m})$ and synergy factor $a$ were determined for native MGD1 and the mutants H155A and P189A, for different lipid monolayer compositions. The uncertainties on MIP and a values were calculated as described in Materials and Methods.

Figure 3: Influence of the lipid nature on the organization of MGD1, H155A and P189A mutants in the membrane.

AFM topographic images of transferred protein/lipids films after protein adsorption on the lipid monolayers at an initial surface pressure $\prod_{i}$ of $15 \mathrm{mN} / \mathrm{m}$. Insets in a, d, g: controls corresponding to the monolayer alone transferred on mica. White arrows in $\mathrm{c}$ and $\mathrm{f}$ indicate dark spots (holes). In all images, scan size is $5 \times 5 \mu \mathrm{m}^{2}$.

Figure 4: Three-dimensional depictions of the macromolecular assembly showing the interaction of MGD1 with a DAG-PG bilayer (1:3 mol/mol) in QuickSurf representation. Color coding: PG (grey), DAG (yellow), N-domain of MGD1 (magenta), C-domain of MGD1 (green), P189 residue (red), H155 residue (blue).

(a) An overall view of the assembly comprising MGD1, 1190 PG, 393 DAG, $28151 \mathrm{H}_{2} \mathrm{O}, 1502$ $\mathrm{Na}^{+}$and $424 \mathrm{Cl}^{-}$. 
(b) View in the membrane plane of the bilayer without protein (left) and with bound MGD1 (right) showing the local concentration of DAG in the vicinity of the protein, which location is indicated by red circle and its orientation in relation to the membrane is given apart.

(c) Two representative orientations of MGD1 with respect to the membrane plane showing the position of $\mathrm{H} 155$ away from the membrane (top) and close to the membrane surface (bottom) as a result of the dynamics of internal motions of C- and N-domains of MGD1.

Figure 5: Proposed model for MGD1 membrane binding and reaction mechanism.

(a) MGD1 is partly embedded in the bilayer through its large and flexible region in the N-domain, which seems essential to the capture of DAG (shown in red) and PG (in blue) molecules.

(b) Schematic representation of MGD1 active site showing the PG-His catalytic dyad capable of deprotonating $\mathrm{OH}$ group of DAG acceptor. Reaction occurs through a $\mathrm{S}_{\mathrm{N}} 2$ mechanism. 